\title{
Interferon Alpha Therapy Increases Pro-Thrombotic Biomarkers in Patients with Myeloproliferative Neoplasms
}

\author{
Dorothée Faille ${ }^{1,2, *(\mathbb{C})}$, Lamia Lamrani ${ }^{1}$, Stéphane Loyau ${ }^{1} \mathbb{C}$, Marie-Geneviève Huisse ${ }^{1,2}$, \\ Marie-Charlotte Bourrienne ${ }^{1,2} \mathbb{D}$, Sawsaneh Alkhaier ${ }^{3}$, Bruno Cassinat ${ }^{3,4}$, Yacine Boulaftali ${ }^{1}$, \\ Jérôme Debus ${ }^{2,5}$, Martine Jandrot-Perrus ${ }^{1}$, Christine Chomienne ${ }^{3,4}$, Christine Dosquet ${ }^{3,4}$ \\ and Nadine Ajzenberg ${ }^{1,2}$ \\ 1 INSERM UMR_S1148, Université de Paris, CEDEX 18, F-75877 Paris, France; lamia.lamrani@hotmail.fr (L.L.); \\ stephane.loyau@inserm.fr (S.L.); marie-genevieve.huisse-ext@aphp.fr (M.-G.H.); \\ marie-charlotte.bourrienne@inserm.fr (M.-C.B.); yacine.boulaftali@inserm.fr (Y.B.); \\ martine.jandrot-perrus@inserm.fr (M.J.-P.); nadine.ajzenberg@aphp.fr (N.A.) \\ 2 Laboratoire d'Hématologie, AP-HP, Hôpital Bichat, CEDEX 18, F-75877 Paris, France; jerome.debus@aphp.fr \\ 3 Service de Biologie Cellulaire, AP-HP, Hôpital Saint Louis, CEDEX 10, F-75475 Paris, France; \\ salkhaeir@ch-valence.fr (S.A.); bruno.cassinat@aphp.fr (B.C.); christine.chomienne@aphp.fr (C.C.); \\ christine.dosquet@aphp.fr (C.D.) \\ 4 INSERM UMR_S1131, Université de Paris, F-75010 Paris, France \\ 5 Laboratoire d'Hématologie, AP-HP, Hôpital Louis Mourier, CEDEX, F-92701 Colombes, France \\ * Correspondence: dorothee.faille@aphp.fr
}

Received: 3 March 2020; Accepted: 11 April 2020; Published: 17 April 2020

\begin{abstract}
Myeloproliferative neoplasms (MPN) are associated with an increased risk of arterial and venous thrombosis. Pegylated-interferon alpha (IFN) and hydroxyurea (HU) are commonly used to treat MPN, but their effect on hemostasis has not yet been studied. The aim of our study was to determine whether IFN and HU impact the biological hemostatic profile of MPN patients by studying markers of endothelial, platelet, and coagulation activation. A total of 85 patients (50 polycythemia vera and 35 essential thrombocythemia) were included: 28 treated with IFN, 35 with $\mathrm{HU}$, and 22 with no cytoreductive drug (non-treated, NT). Von Willebrand factor, shear-induced platelet aggregation, factor VIII coagulant activity (FVIII:C), fibrinogen, and thrombin generation with and without exogenous thrombomodulin were significantly higher in IFN-treated patients compared to NT patients, while protein $S$ anticoagulant activity was lower. In 10 patients in whom IFN therapy was discontinued, these hemostatic biomarkers returned to the values observed in NT patients, strongly suggesting an impact of IFN therapy on endothelial and coagulation activation. Overall, our study shows that treatment with IFN is associated with significant and reversible effects on the biological hemostatic profile of MPN patients. Whether they could be associated with an increased thrombotic risk remains to be determined in further randomized clinical studies.
\end{abstract}

Keywords: myeloproliferative neoplasms; interferon; prothrombotic markers

\section{Introduction}

Myeloproliferative neoplasms (MPN) are clonal diseases resulting from the dysregulated production of mature myeloid cells by hematopoietic stem cells affected by a molecular defect. BCR-ABL-negative MPN include polycythemia vera (PV), essential thrombocytemia (ET), and primary myelofibrosis. They are associated with somatic mutations in 3 driver genes: JAK2, CALR, and MPL in more than $90 \%$ of patients. The most frequent one is JAK2V617F mutation [1]. Irrespective to the 
mutation, the hyperactivation of JAK2-STAT signaling has a central role in MPN pathophysiology [2,3]. MPN are characterized by a predisposition to thrombotic events that significantly affect patient morbidity and mortality. Thrombotic events at diagnosis and during follow up are more frequent in PV than in ET, with the prevalence of major thrombosis at diagnosis ranging from $19 \%$ to $38 \%$ and from $7 \%$ to $26 \%$, respectively [4]. In both PV and ET, arterial thrombosis accounts for approximately two-thirds of events and consists mainly in ischemic stroke and in myocardial infarction. Deep vein thrombosis, pulmonary embolism, and splanchnic vein thrombosis are the most frequent localizations of venous events. The pathogenesis of thrombosis is assumed to be multifactorial. An increased number and abnormal functions of blood cells both contribute to the prothrombotic phenotype. Neoplastic cells produce cytokines that are responsible for the proinflammatory phenotype of vascular endothelial cells, contributing to thrombotic risk [5]. JAK2V617F expression by endothelial cells could also alter their phenotype [6]. Thus, numerous studies have provided evidence that the pathogenesis of MPN-associated thrombosis involves the activation of platelets (including the production of procoagulant microvesicles), of neutrophils (including the release of proteolytic enzymes and reactive oxygen species), of red blood cells and of endothelial cells [7].

Risk stratification in PV and ET has been designed to estimate the likelihood of thrombotic complications [8]. Predictors of arterial complications include age $>60$ years, leukocytosis $>11 \mathrm{G} / \mathrm{L}$, prior history of thrombosis, and cardiovascular risk factors [9]. High-risk PV and ET patients require cytoreductive therapy, while low-risk patients require daily aspirin therapy [8]. Treated PV patients with hematocrit less than $45 \%$ have a lower risk of cardiovascular death and major thrombosis than patients with higher hematocrit [10]. The first-line drug of choice for cytoreductive therapy, in both PV and $\mathrm{ET}$, is hydroxyurea (HU), while the second-line drugs of choice include pegylated-interferon alpha (IFN) [8].

The IFN mechanism of action in MPN has not been completely elucidated. IFN has a direct pro-apoptotic effect on myeloid progenitors and more particularly on erythroid progenitors in humans as well as in mouse models. A direct anti-proliferative effect of IFN on stem cells has also been suggested as well as an immunomodulator effect that enhances the host immune response and restores efficient immune surveillance. However, there is no clear demonstration of such mechanisms in human MPN [11]. Interestingly, Prendergast and colleagues described that IFN treatment of mice led to a rapid stimulation of bone marrow endothelial cells in vivo [12].

Unexpectedly, HU, but not IFN, was recently reported to enhance the expression of adhesion molecules at the surface of red blood cells from PV patients, resulting in increased adhesion to laminin [13]. Thus, it is important to better characterize the effect of both drugs with respect to the activation of blood cells and coagulation.

Therefore, we evaluated biological markers of coagulation and endothelial activation in MPN patients who were treated by IFN compared to patients treated by $\mathrm{HU}$ and to patients with no cytoreductive drugs at the moment of inclusion.

\section{Results}

\subsection{Patient Characteristics}

Demographic, clinical, and biological characteristics of patients are depicted in Table 1. Among the 85 patients (50 PV and $35 \mathrm{ET}$ ), 22 were not treated with any cytoreductive drug (NT) at the inclusion, 35 were treated by HU and 28 by IFN. HU-treated patients were older than NT and IFN-treated patients $(p=0.001$ and 0.0002 , respectively). PV was more frequent in HU-and IFN-treated patients than in the NT group $(p=0.002)$. Median time from diagnosis to inclusion was 7 years in NT group, 3 years in HU-treated group, and 10.5 years in IFN-treated group. Cytoreductive therapy had been initiated at diagnosis in $23 / 35(65.7 \%)$ and in $8 / 28(28.6 \%)$ patients from HU- and IFN-treated groups, respectively. Median time from initiation of treatment to inclusion in the study was not statistically different between patients treated with HU or IFN. All these patients were treated for at least 4 months and were 
receiving aspirin when included. The median cumulative dose of IFN in IFN-treated patients was 10,800 $\mu \mathrm{g}$ (interquartile range, IQR: 6480-16,470). Four patients with PV were treated by phlebotomy. Among them, one was also treated by HU. The occurrence of previous thrombotic events (arterial and venous) was similar between the 3 groups of patients. Bleeding episodes such as epistaxis or bruises were not statistically different according to treatment. Frequency of the JAK2V617F mutation was in agreement with previous published data (51\% in ET and 98\% in PV patients) (Table S1) [1,14] and did not differ among the NT, HU-treated, and IFN-treated patients. JAK2V617F burden was not statistically different among the 3 groups of patients according to treatment (mean \pm SD: $19 \pm 20 \%, 34 \pm 22 \%$, and $29 \pm 25 \%$ in NT, HU-treated, and IFN-treated patients, respectively). As expected, platelet count was higher in ET compared to PV patients $(p<0.0001)$, and hematocrit and hemoglobin were higher in PV compared to ET patients ( $p=0.001$ and $p=0.01$, respectively) (Table S1). Blood cell counts were higher in NT patients compared to treated patients. Platelet count was higher in NT patients compared to HU- and IFN-treated patients ( $p=0.007$ and $p=0.0003$, respectively). HU-treated patients had also a significant higher platelet count than IFN-treated patients $(p=0.005)$. Hemoglobin was higher in NT and HU-treated patients compared to IFN-treated patients ( $p=0.01$ and $p=0.03$, respectively) and leukocyte count was significantly higher in NT and HU-treated patients compared to IFN-treated patients ( $p=0.0001$ and $p=0.0003$, respectively).

Table 1. Demographic, clinical, and biological characteristics of the study population.

\begin{tabular}{|c|c|c|c|c|}
\hline Characteristics & $\begin{array}{c}\mathrm{NT} \\
(n=22)\end{array}$ & $\begin{array}{c}\text { HU } \\
(n=35)\end{array}$ & $\begin{array}{c}\text { IFN } \\
(n=28)\end{array}$ & $p$-Value \\
\hline Age, years & $55.5(50-68.5)$ & $72(62-78)$ & $58.5(50-66.5)$ & 0.0001 \\
\hline Gender, male & $14(64)$ & $16(46)$ & $9(32)$ & 0.08 \\
\hline \multicolumn{5}{|l|}{ Disease type } \\
\hline PV & $6(27)$ & $24(69)$ & $20(71)$ & 0.002 \\
\hline ET & $16(73)$ & $11(31)$ & $8(29)$ & \\
\hline \multicolumn{5}{|l|}{ Time, years: } \\
\hline from diagnosis to inclusion & $7(1-12)$ & $3(1-11)$ & $10.5(5-21.5)$ & 0.007 \\
\hline from diagnosis to treatment & NA & $0(0-1)$ & $7.5(1-17.5)$ & $<0.0001$ \\
\hline from treatment to inclusion & NA & $3(0-6)$ & $2(1.5-3)$ & 0.5 \\
\hline \multicolumn{5}{|l|}{ Aspirin dose } \\
\hline $75 \mathrm{mg}$ & $10(45.5)$ & $24(68.5)$ & $22(79)$ & 0.1 \\
\hline $100 \mathrm{mg}$ & $11(50)$ & $9(26)$ & $5(18)$ & \\
\hline $160 \mathrm{mg}$ & $1(4.5)$ & $2(5.5)$ & $1(3)$ & \\
\hline \multicolumn{5}{|l|}{ History of thrombosis } \\
\hline Arterial & $6(27)$ & $8(23)$ & $4(14)$ & 0.5 \\
\hline Venous & $2(9)$ & $4(11)$ & $1(3)$ & 0.5 \\
\hline History of bleeding & $0(0)$ & $5(14)$ & $7(25)$ & 0.04 \\
\hline \multicolumn{5}{|l|}{ Mutations } \\
\hline JAK2V617F & $15(68)$ & $29(83)$ & $23(82)$ & 0.4 \\
\hline CALR & $3(14)$ & $5(14)$ & $3(11)$ & \\
\hline JAK2 exon 12 & $1(4)$ & $0(0)$ & $0(0)$ & \\
\hline MPL & $0(0)$ & $0(0)$ & $1(3.5)$ & \\
\hline Triple negative & $3(14)$ & $1(3)$ & $1(3.5)$ & \\
\hline \multicolumn{5}{|l|}{ Blood cell counts } \\
\hline Platelets, $10^{9} / \mathrm{L}$ & $552(265-732)$ & $324(242-437)$ & $194(151-323)$ & 0.0001 \\
\hline Hemoglobin, g/dL & $14.7(13.4-15.8)$ & 14.5 (13.7-15.7) & $13.7(13-14.5)$ & 0.03 \\
\hline Hematocrit, \% & $44.1(39.3-46.1)$ & $44.1(40.9-46.2)$ & $41.0(38.1-43.0)$ & 0.048 \\
\hline Leukocytes, $10^{9} / \mathrm{L}$ & $6.8(5.9-8.6)$ & $6.1(5.1-7.5)$ & $4.5(3.3-5.9)$ & $<0.0001$ \\
\hline
\end{tabular}

ET: essential thrombocythemia, HU: hydroxyurea, IFN: interferon-treated patients, NA: not applicable, NT: not treated with a cytoreductive drug at the inclusion, PV: polycythemia vera. Results are presented as number (percentage) for categorical variables or as median (IQR) for continuous variables; $p$-value for Chi-square, Mann-Whitney or Kruskal-Wallis test. 


\subsection{Markers of Endothelial Activation Are Increased in IFN-Treated Patients}

To analyze the effect of IFN treatment on the activation of the vascular endothelium, we measured the levels of von Willebrand factor (vWF) that is released from endothelial cells upon activation. vWF antigen was higher in IFN-treated patients compared to NT and HU-treated patients $(p<0.0001$ and $p=0.004$, respectively) (Table 2 and Figure 1A). The same results were observed for and vWF activity (Table 2 and Figure 1B). In a logistic regression analysis, these results remained significant after adjustment for age, sex, and disease type (ET or PV). In HU-treated patients, vWF antigen and activity were also significantly higher compared to NT patients ( $p=0.002$ and $p=0.03$, respectively). No correlation could be observed between IFN dose and vWF antigen or activity. As platelet aggregation under high shear conditions is dependent of vWF, we evaluated shear-induced platelet aggregation (SIPA) at $4000 \mathrm{~s}^{-1}$ in all patients. SIPA was higher in IFN-treated patients compared to NT and HU-treated patients ( $p=0.0005$ and $p=0.002$, respectively), independently of age, sex, and disease type. No difference was observed between HU-treated and NT patients (Table 2 and Figure 1C).

Table 2. Biomarkers of endothelial activation according to cytoreductive treatment group.

\begin{tabular}{ccccc}
\hline Biomarkers & $\begin{array}{c}\text { NT } \\
(\boldsymbol{n}=\mathbf{2 2})\end{array}$ & $\begin{array}{c}\text { HU } \\
(\boldsymbol{n}=\mathbf{3 5})\end{array}$ & $\begin{array}{c}\text { IFN } \\
(\boldsymbol{n}=\mathbf{2 8})\end{array}$ & $\boldsymbol{p}$-Value \\
\hline vWF antigen, \% & $101(70-143)$ & $161(113-202)$ & $201(158-298)$ & $<0.0001$ \\
vWF activity, \% & $103(64-141)$ & $139(104-168)$ & $178(141-240)$ & 0.0001 \\
SIPA, \% * & $10.6(2.0-16.7)$ & $13.0(5.8-23.5)$ & $30.4(13.0-46.1)$ & 0.0004 \\
\hline
\end{tabular}

ET: essential thrombocythemia, HU: hydroxyurea, IFN: interferon-treated patients, NT: not treated with a cytoreductive drug at the inclusion, PV: polycythemia vera, SIPA: shear-induced platelet aggregation, vWF: von Willebrand factor. Results are presented as median (IQR); $p$-value for Kruskal-Wallis test. * Due to missing points, SIPA results from 19 NT and 27 IFN-treated patients are presented.

A.

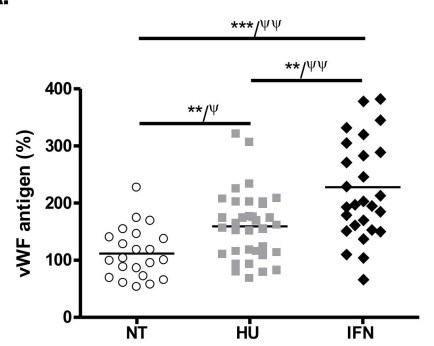

B.

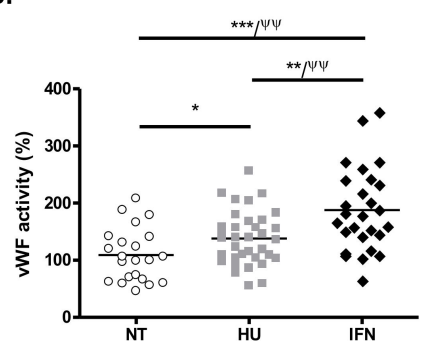

c.

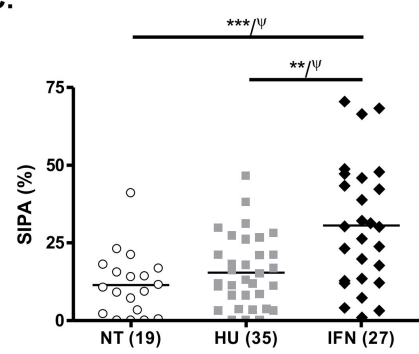

Figure 1. Von Willebrand factor antigen (A), von Willebrand factor activity (B), and platelet aggregation induced by high shear at $4000 \mathrm{~s}^{-1}$ (SIPA, C) in NT, HU-, and IFN-treated patients. Post-hoc pairwise comparisons of parameters were performed using Mann-Whitney test $\left({ }^{*} p<0.05,{ }^{* *} p<0.01,{ }^{* * *} p<0.001\right)$ and logistic regression analysis adjusted on age, sex, and disease type (ET or PV) $\left({ }^{\Psi} p<0.05,{ }^{\Psi \Psi} p<0.01\right)$.

\subsection{IFN Treatment Does Not Activate Platelets In Vivo}

To analyze the effect of IFN treatment on platelet activation in vivo, the exposure of P-selectin, a granule secretion marker, and activated GPIIbIIIa at the platelet surface was measured. No difference was observed between the 3 groups of patients, and levels remained in the normal ranges (Table 3). To further examine platelet activation status, we determined the level of circulating platelet-monocyte aggregates (PMA) and platelet-neutrophil aggregates (PNA) in the 3 groups of patients according to treatment. Percentages of aggregates remained in the normal ranges and did not differ among the 3 groups. 
Table 3. Markers of in vivo platelet activation according to cytoreductive treatment group.

\begin{tabular}{ccccc}
\hline Markers & $\begin{array}{c}\text { NT } \\
(\boldsymbol{n}=\mathbf{2 2})\end{array}$ & $\begin{array}{c}\text { HU } \\
(\boldsymbol{n}=\mathbf{3 5 )}\end{array}$ & $\begin{array}{c}\text { IFN } \\
(\boldsymbol{n}=\mathbf{2 8})\end{array}$ & $\boldsymbol{p}$-Value \\
\hline P-selectin, \% & $1.2(0.4-1.6)$ & $1.2(0.4-2.0)$ & $0.7(0.3-1.6)$ & 0.6 \\
Activated GpIIbIIIa, \% & $0.4(0.1-2.8)$ & $1.8(0.6-5.6)$ & $1.2(0.4-3.4)$ & 0.055 \\
PMA, \% & $6.8(5.2-10.2)$ & $9.0(6.4-15.0)$ & $8.0(6.4-9.4)$ & 0.2 \\
PNA, \% & $7.8(6.3-10.0)$ & $9.4(7.6-11.7)$ & $7.5(6.4-9.2)$ & 0.08 \\
\hline
\end{tabular}

ET: essential thrombocythemia, HU: hydroxyurea, IFN: interferon-treated patients, NT: not treated with a cytoreductive drug at the inclusion, PMA: platelet-monocyte aggregates, PNA: platelet-neutrophil aggregates, PV: polycythemia vera. Results are presented as median (IQR); $p$-value for Kruskal-Wallis test.

\subsection{IFN Treatment Does Not Modify Biological Response to Aspirin Treatment}

Since aspirin could influence the profile of platelet activation markers, we verified the compliance of patients and tested the effectiveness of aspirin in inhibiting arachidonic acid-induced platelet aggregation. A total of 12 patients were not evaluable, because of too recent aspirin intake. Biological efficacy of aspirin was not different between the 3 groups of patients according to treatment (Table S2).

\subsection{IFN-Treated Patients Display A Hypercoagulable Profile}

To investigate the hemostatic balance between anti- and pro-coagulant parameters, we first measured pro-coagulant factors such as factor VIII coagulant activity (FVIII:C) and fibrinogen, and anticoagulant proteins such as protein S, protein $\mathrm{C}$, and antithrombin. FVIII:C was significantly higher in IFN-treated patients compared to NT and HU-treated patients $(p=0.003$ and $p<0.0001$, respectively) (Table 4 and Figure 2A). Furthermore, fibrinogen was slightly but significantly higher in IFN-treated patients compared to NT patients $(p=0.004)$ (Table 4 and Figure 2B). Conversely, protein S was significantly lower in IFN-treated patients compared to NT patients $(p<0.0001)$ and to HU-treated patients $(p<0.0001)$ (Table 4 and Figure 2C). In a logistic regression analysis, these results remained significant after adjustment for age, sex, and disease type. Levels of protein $C$ and antithrombin were in the normal range without difference among the 3 groups of patients (Table 4).

A.

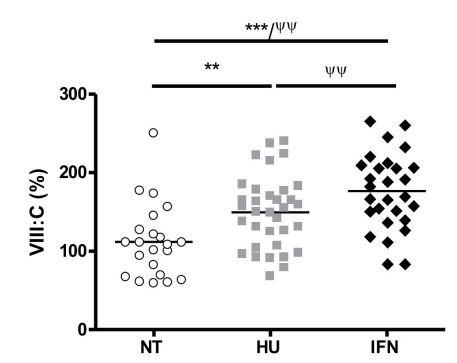

B.

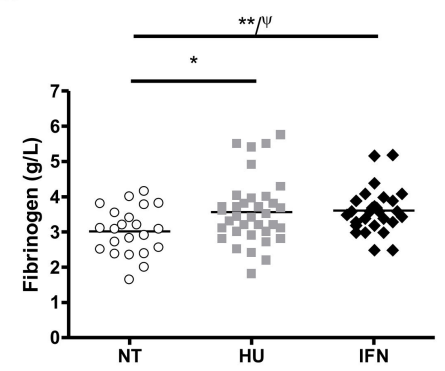

c.

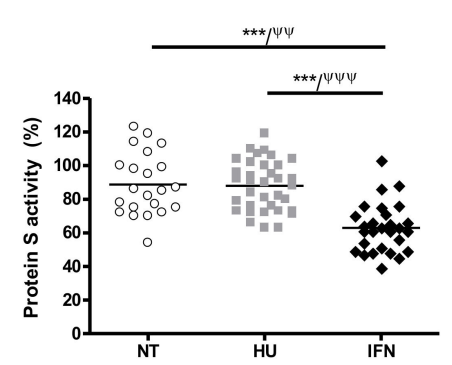

Figure 2. Levels of VIII:C (A) fibrinogen (B) and protein S activity (C) in NT, HU- and IFN-treated patients. Post-hoc pairwise comparisons of parameters were performed using the Mann-Whitney test $\left({ }^{*} p<0.05,{ }^{* *} p<0.01,{ }^{* * *} p<0.001\right)$ and logistic regression analysis adjusted for age, sex, and disease type (ET or PV) $\left({ }^{\Psi} p<0.05,{ }^{\Psi \Psi} p<0.01,{ }^{\Psi \Psi \Psi} p<0.001\right)$. 
Table 4. Pro- and anti-coagulant factors according to cytoreductive treatment group.

\begin{tabular}{ccccc}
\hline Factors & $\begin{array}{c}\text { NT } \\
(\boldsymbol{n}=\mathbf{2 2})\end{array}$ & $\begin{array}{c}\text { HU } \\
(\boldsymbol{n}=\mathbf{3 5 )}\end{array}$ & $\begin{array}{c}\text { IFN } \\
(\boldsymbol{n}=\mathbf{2 8})\end{array}$ & $\boldsymbol{p}$-Value \\
\hline VIII:C, \% & $109(68-136)$ & $155(107-178)$ & $176(145-208)$ & $<0.0001$ \\
Fibrinogen, g/dL & $3.1(2.4-3.6)$ & $3.4(3.0-3.9)$ & $3.6(3.2-3.9)$ & 0.02 \\
Protein S, \% & $85(73-104)$ & $90(73-100)$ & $62(50-70)$ & $<0.0001$ \\
Protein C, \% & $103(96-123)$ & $124(101-130)$ & $117(93-128)$ & 0.4 \\
Antithrombin, \% & $108(101-111)$ & $106(101-118)$ & $111(103-119)$ & 0.3 \\
\hline
\end{tabular}

ET: essential thrombocythemia, HU: hydroxyurea, IFN: interferon-treated patients, NT: not treated with a cytoreductive drug at the inclusion, PV: polycythemia vera. Results are presented as median (IQR); $p$-value for Kruskal-Wallis test.

Next, we used a global thrombin generation assay with or without the addition of recombinant thrombomodulin in plasma. In the absence of thrombomodulin, the thrombin peak (Table 5 and Figure 3A) and velocity index (Table 5 and Figure 3C) were significantly higher in HU- $(p=0.02)$ and in IFN-treated patients ( $p=0.03$ and $p=0.04$, respectively) compared to NT patients. In a logistic regression analysis, these results were not significantly different anymore, suggesting that thrombin generation results were dependent of age, sex, and/or disease type. Neither thrombin peak nor velocity differed between HU- and IFN-treated patients. No difference was evidenced for the endogenous thrombin potential (ETP) among the 3 groups (Table 5 and Figure 3B). As expected, adding thrombomodulin to the plasma markedly inhibited thrombin generation as indicated by the lower peak and ETP values (Table 5 and Figure 3D-E) compared to the results obtained in the absence of thrombomodulin. In the presence of thrombomodulin, thrombin peak (Table 5 and Figure 3D), ETP (Table 5 and Figure 3E), and velocity index (Table 5 and Figure 3F) were significantly higher in HU- ( $p=0.02, p=0.04$ and $p=0.02$, respectively) and in IFN-treated patients $(p=0.01, p=0.02$ and $p=0.01$, respectively) compared to NT patients. No difference was evidenced between HU- and IFN-treated patients (Table 5 and Figure 3D-F).

A.

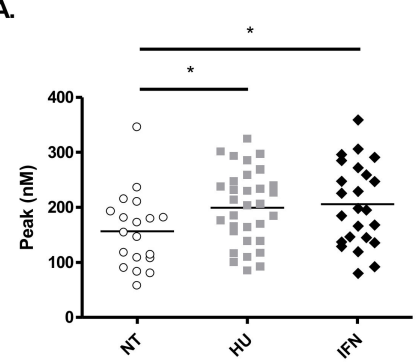

D.

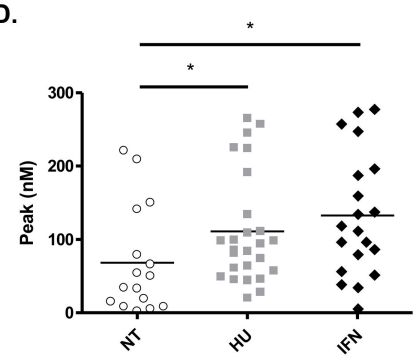

B.

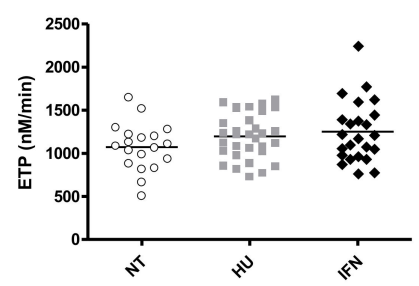

E.

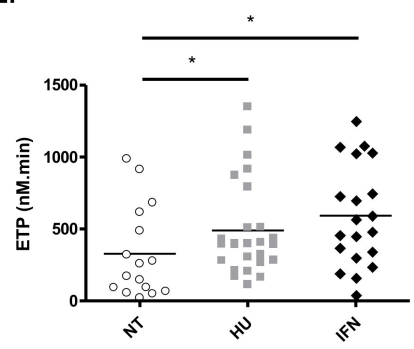

c.

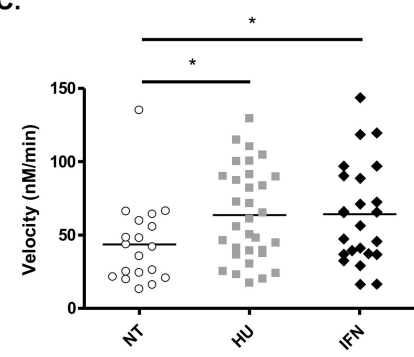

F.

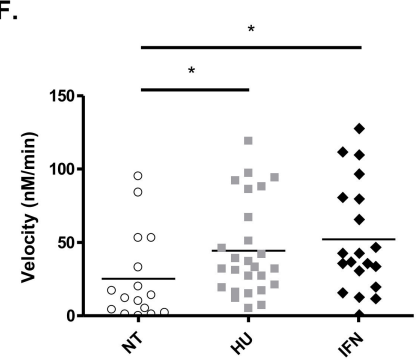

Figure 3. Thrombin generation in the plasma of NT, HU- and IFN-treated patients in the absence (A-C) and in the presence (D-F) of thrombomodulin. Peak of thrombin (A,D), ETP (B,E), and velocity $(\mathbf{C}, \mathbf{F})$. Post-hoc pairwise comparisons of parameters were performed using the Mann-Whitney test. ${ }^{*} p<0.05$. 
Table 5. Parameters of thrombin generation according to cytoreductive treatment group.

\begin{tabular}{ccccc}
\hline Parameters & $\begin{array}{c}\text { NT } \\
(n=22)\end{array}$ & $\begin{array}{c}\text { HU } \\
(n=35)\end{array}$ & $\begin{array}{c}\text { IFN } \\
(n=28)\end{array}$ & $p$-Value \\
\hline Without thrombomodulin & & & & \\
Peak, nM & $154(107-192)$ & $205(147-243)$ & $197(142-266)$ & 0.04 \\
ETP, nM.min & $1080(879-1218)$ & $1215(1021-1428)$ & $1194(975-1422)$ & 0.2 \\
Velocity, nM/min & $42(21-60)$ & $58(38-90)$ & $61(37-90)$ & 0.049 \\
With thrombomodulin & & & & \\
Peak, nM & $42(11-110)$ & $89(53-162)$ & $115(68-192)$ & 0.02 \\
ETP, nM.min & $215(78-552)$ & $397(274-653)$ & $525(321-887)$ & 0.03 \\
Velocity, nM/min & $13.0(3.0-43.0)$ & $32(18-76)$ & $40(25-80)$ & 0.02 \\
\hline
\end{tabular}

ET: essential thrombocythemia, ETP: endogenous thrombin potential, HU: hydroxyurea, IFN: interferon-treated patients, NT: not treated with a cytoreductive drug at the inclusion, PV: polycythemia vera. Results are presented as median (IQR); $p$-value for Kruskal-Wallis test.

\subsection{Discontinuation of IFN Treatment Restores Hemostatic Parameters to Normal Levels}

To further validate our hypothesis that the elevation of plasma pro-coagulant biomarkers in IFN-treated patients was directly related to IFN therapy, we took advantage of the discontinuation of IFN treatment in some patients. A total of 10/28 IFN-treated patients (5 ET and $5 \mathrm{PV}$ ) discontinued IFN treatment because of side effects $(n=4)$, hematological or molecular response $(n=5)$, or personal preference $(n=1)$.

Biological parameters were tested at least 6 months after IFN discontinuation. Interestingly, vWF antigen and activity, FVIII:C, fibrinogen, and protein $\mathrm{S}$ activity returned to levels similar to those observed in NT patients and were significantly different from levels observed during IFN treatment. vWF antigen decreased significantly from $182 \%(153-273 \%)$ to $92 \%(78-104 \%)(p=0.002)$, vWF activity from $172 \%(135-228 \%$ ) to $92 \%$ (82-128\%) ( $p=0.002)$, FVIII:C from $158 \%(128-217 \%)$ to $112 \%(74-141 \%)$ $(p=0.004)$, fibrinogen from $3.7(3.4-4.6)$ to $3.1(2.8-3.4) \mathrm{g} / \mathrm{L}(p=0.004)$, and protein $\mathrm{S}$ activity increased from $62 \%(48-75 \%)$ to $87 \%(77-111 \%)(p=0.002)$ (Figure $4 \mathrm{~A}-\mathrm{F})$.

A.

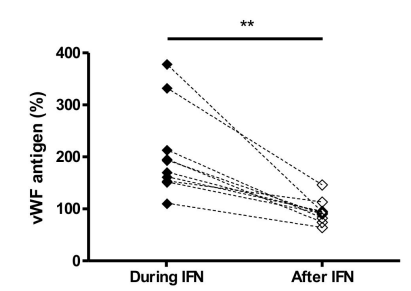

D.

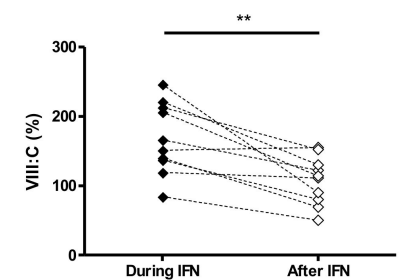

B.

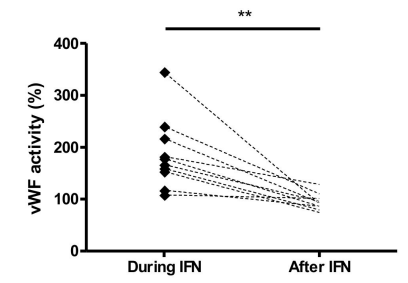

E.

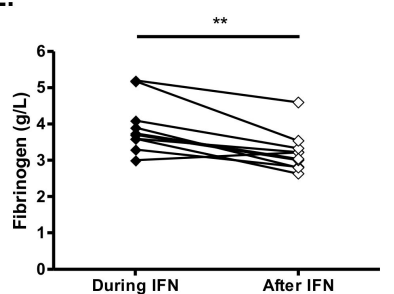

c.

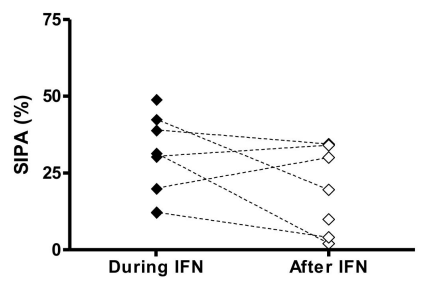

F.

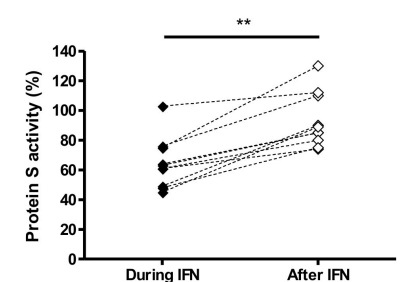

Figure 4. von Willebrand factor antigen (A), von Willebrand factor activity (B), platelet aggregation induced by high shear at $4000 \mathrm{~s}^{-1}$ (SIPA, C), VIII:C (D) fibrinogen (E) and protein S activity (F) during IFN treatment and after IFN discontinuation in 10 patients. Comparison between the 2 groups was performed using Wilcoxon matched pairs test. ${ }^{* *} p<0.01$. 


\section{Discussion}

This observational single-center cohort study aimed to evaluate the impact of IFN and HU on the biological hemostatic profile of MPN patients. Here, we report that IFN therapy, and to a lesser extent HU treatment, are associated with significantly higher levels of pro-thrombotic markers compared to NT patients, independently of age, sex, and disease type. Most clinical studies have reported a complete and sustained molecular response in 35\% of MPN patients after IFN administration [11,15], but there is currently no clinical data on the potential side effects of IFN on hemostasis.

IFN alpha is able to activate the MAP kinase pathway responsible for inflammation [16]. It has been demonstrated that there is a synergistic effect of IFN alpha and Toll-like receptor 4 ligands in the development of atherosclerotic plaque [17]. In mouse models, IFN alpha treatment led to a rapid stimulation of bone marrow endothelial cells in vivo [12], promoting inflammation. In the present study, we show that vWF antigen and activity were significantly higher in IFN-treated patients than in NT patients. These results are consistent with those reported in patients treated by IFN for hepatitis, who presented similarly higher vWF levels, suggesting an activation of endothelial cells in vivo [18].

SIPA in high shear rate conditions is able to reproduce stenotic arteries and is usually correlated to vWF level [19]. Consistent with the high levels of vWF found in our study, SIPA was also higher in IFN-treated patients. We also evaluated platelet activation markers. The exposure of P-selectin and activated GPIIbIIIa as well as platelet-leucocyte aggregate levels remained low and were similar among groups. Since the frequency of biological resistance to aspirin was comparable among the 3 treatment groups, we can assume that these results were not influenced by aspirin. Overall, these data are not in favor of in vivo platelet activation in our cohort and are discrepant with data from other studies reporting elevated markers of platelet activation in MPN patients [20]. This could be explained by two hypotheses: (i) NT patients had a low level of disease progression and (ii) cytoreductive therapy was efficient in the treated groups.

Aside from vWF, FVIII is the only coagulation factor upstream of thrombin generation that was higher in IFN-treated patients and to a lesser extent in HU-treated patients compared to NT patients, probably because FVIII circulates associated to vWF. Interestingly, fibrinogen level was also significantly higher in these patients, suggesting an inflammatory state. However, C-reactive protein was not statistically different among groups, which was probably because of its shorter half-life as compared to fibrinogen. We reported an elevation of thrombin generation in HU- and IFN-treated patients compared to NT patients. Similar results were observed in a previous study comparing PV, ET, and controls [21]. In two other studies, a decrease in thrombin generation in MPN patients compared to controls was observed but with much higher tissue factor concentrations (up to $6.8 \mathrm{pM}$ ) [22,23]. In order to assess the effectiveness of the protein C/protein $S$ anticoagulant pathway, thrombomodulin was added to the thrombin generation assay [24]. The elevation of thrombin generation in HU- and IFN-treated patients was even more significant in the presence of thrombomodulin. This could be explained by the reduced levels of free protein $S$ as previously reported in a global coagulation study in MPN patients compared to controls [25]. These data suggest a pro-coagulant imbalance in IFN-treated patients compared to HU-treated and NT patients, even though we could not find any correlation between protein $S$ levels and thrombin generation parameters in the presence of thrombomodulin, which was possibly due to the limited number of patients studied.

Importantly, we found that vWF antigen and activity, protein S activity, FVIII:C, and fibrinogen returned to levels observed in NT patients, when patients interrupted IFN treatment for at least 6 months. These data support the causal effect of IFN treatment in the modification of these biological parameters and thus in the generation of a potential pro-thrombotic tendency.

This study has certain limitations in addition to the limited number of patients included. First, the patients of our cohort were in a steady state explaining why some markers such as platelet activation markers were not different between groups, in contrast to previous studies [20-23,26]. Moreover, treatment by aspirin in all patients could have minimized their platelet activation profile. Second, as only two patients presented thrombotic events after treatment initiation, the association between 
thrombosis and treatment could not be established. Finally, the low number of IFN-treated patients with calreticulin (CALR) mutation did not allow for subgroup analysis of pro-thrombotic biomarkers according to the mutational status in IFN-treated patients.

\section{Materials and Methods}

\subsection{Patients}

Between January 2012 and February 2014, 85 patients referred to the Unit of Cellular Biology at Saint-Louis hospital in Paris were enrolled in this study. PV and ET patients were diagnosed according to WHO criteria. Blood samples were collected in the Department of Biological Hematology in Bichat hospital. The INSERM Ethics Evaluation Committee (CEEI/IRB) approved the protocol (IRB00006477, opinion number 11-106). All patients provided a written informed consent. At the moment of inclusion, patients were receiving treatment with HU or IFN for at least 4 months or were not treated with any cytoreductive drug. None of the patients was taking anticoagulant or antiplatelet therapy other than aspirin.

\subsection{Genotyping MPN Mutations}

JAK2V617F mutation was detected using the Ipsogen JAK2 MutaQuant kit (Qiagen, Courtaboeuf, France) according to manufacturer's instructions. A high resolution melting (HRM) method was used to detect JAK2 exon 12 mutations as previously described [27]. Screening for the presence of CALR mutations was performed as previously described [28]. Finally, p.W515L and p.W515K MPL mutations were detected using Ipsogen MPL W515L/K MutaScreen kit (Qiagen, Courtaboeuf, France) according to the manufacturer's instructions.

\subsection{Platelet Rich and Poor Plasma Preparation}

Blood samples were collected in vacuum tubes (Greiner-Bio One, Les Ulis, France) containing citrate $(0.109 \mathrm{M})$ for platelet and coagulation studies or EDTA for blood cell count. Platelet-rich plasma (PRP) was obtained by centrifugation of whole blood at $120 \mathrm{~g}$ for $15 \mathrm{~min}$ at room temperature. Platelet-poor plasma (PPP) was isolated by the double centrifugation of blood samples at $2500 \mathrm{~g}$ for $15 \mathrm{~min}$ at $18{ }^{\circ} \mathrm{C}$ and stored at $80^{\circ} \mathrm{C}$ until further analysis.

\subsection{Von Willebrand Factor (vWF) Measurements}

vWF activity was determined by vWF Ristocetin Cofactor (vWF:RCo) assay using light transmission aggregometry (ChronoLog Havertown, PA, USA). PPP was incubated with lyophilized platelets (Hyphen BioMed, Neuville sur Oise, France) and ristocetin (1.2 mg/mL) (Diagnostica Stago, Asnières, France). Results were expressed as percentage of vWF activity compared to reference plasma. vWF antigen was measured by ELISA (Asserachrom vWF antigen, Diagnostica Stago) following manufacturer's instructions.

\subsection{Shear-Induced Platelet Aggregation (SIPA)}

Shear experiments were performed on PRP by means of a coaxial cylinder-shearing device at $4000 \mathrm{~s}^{-1}$ as previously described [29]. SIPA was measured on an EPICS XL flow cytometer (Beckman Coulter, Villepinte, France). The results of platelet aggregation were expressed as percentage of disappearance of single platelets (DSP): DSP $=\left[\left(n_{0}-n\right) / n_{0}\right] \times 100$, when $n_{0}$ represents the single platelet population of non-sheared control sample and $n$ represents that of the sheared sample.

\subsection{Coagulation Parameter Measurements}

Fibrinogen (Dade Thrombin reagent, Siemens, Marburg, Germany), factor VIII coagulant (FVIII:C) (Factor VIII deficient plasma, Siemens), protein S activity (Staclot Protein S, Diagnostica Stago), 
protein C anticolagulant activity (Cryocheck Clot C, Cryopep, Montpellier, France), and antithrombin (Stachrom AT III, Diagnostica Stago) were measured on a STA-R Analyser (Diagnostica Stago).

\subsection{Platelet Activation Markers}

In vivo platelet activation was evaluated by measuring the surface expression of P-selectin (CD62P) and activated GPIIb/GPIIIa (PAC-1) by flow cytometry. Briefly, diluted whole blood was incubated with fluorescein isothiocyanate (FITC)-conjugated anti-CD62P or PAC-1 (Beckman Coulter, Villepinte, France) for $30 \mathrm{~min}$ in the dark. Samples were analyzed with an EPICS XL flow cytometer. Platelets were gated on forward and side scatter and the percentage of P-selectin and PAC-1-positive platelets were obtained after subtracting the percentage of positive platelets obtained with the FITC-conjugated isotype control for CD62P (IgG1) and PAC-1 (IgM), respectively.

\subsection{Platelet-Leukocyte Complexes}

Whole-blood platelet-leukocyte complexes were counted by flow cytometry. Briefly, PMA were defined as the proportion of monocytes labeled with anti-CD14-FITC (Beckman Coulter) positive for phycoerythrin (PE)-conjugated anti-CD41 (Beckman Coulter) and PNA were defined as the proportion of neutrophils labeled with phycoerythrin-cyanine 5(PC5)-conjugated anti-CD45 (Beckman Coulter) positive for anti-CD41-PE. Samples were analyzed with an EPICS XL flow cytometer.

\subsection{Thrombin Generation Assay}

Thrombin generation was measured on PPP using calibrated automated thrombogram (CAT, Diagnostica Stago) as previously described [30]. In PPP, thrombin generation was triggered by $1 \mathrm{pM}$ tissue factor and $4 \mathrm{mM}$ phospholipids (PPP-Reagent LOW, Diagnostica Stago) in the absence or in the presence of rabbit thrombomodulin (American Diagnostica, $4 \mathrm{nM}$ ). The following parameters were quantified: peak (maximal thrombin concentration, in $\mathrm{nM}$ of thrombin), velocity (slope of thrombin generated in $\mathrm{nM}$ of thrombin/min), and ETP (total amount of thrombin generated, in $\mathrm{nM}$ of thrombin/min).

\subsection{Evaluation of Biological Efficacy of Aspirin}

Arachidonic acid-induced platelet aggregation was studied in PRP by light transmittance aggregometry after the addition of $1.25 \mu \mathrm{M}$ arachidonic acid (Chrono-Par, Chrono-Log corp. Havertown, PA, USA). Resistance to aspirin was defined by maximal platelet aggregation $>20 \%$ tested at trough level just before the daily intake of aspirin. Platelet count in PRP was adjusted to $600 \mathrm{G} / \mathrm{L}$ with PPP if the platelet count in native PRP was above.

\subsection{Statistical Analysis}

Categorical variables are presented as number with percentage. Continuous variables were tested for normality distribution with the Kolmogorov-Smirnov test and are expressed median with IQR (25th-75th percentiles). Comparisons among the 3 groups were performed using Kruskal-Wallis test for continuous variables and Chi-square test for categorical variables. Post-hoc pairwise comparison of parameters was performed using the Mann-Whitney test and logistic regression analysis was used to adjust for age, sex, and disease type (PV or ET). A comparison of biological parameters during IFN treatment and after IFN discontinuation was performed with the Wilcoxon matched pairs test. Analysis of correlation was performed using Spearman's rank correlation coefficient. For all of the statistical analyses, a value of $p<0.05$ was considered statistically significant. GraphPad Prism 5 (GraphPad Software) was used for all analyses. 


\section{Conclusions}

Overall, our study shows that the treatment with IFN is associated with significant and reversible effects on the biological hemostatic profile of MPN patients. The elevation of pro-thrombotic biomarkers in MPN patients treated with IFN should be considered at least a biological side effect of this treatment. Thus, our study points out that caution should be taken and further preventive antithrombotic therapy other than aspirin alone could be required in IFN-treated patients with additional common thrombotic risk factors such as FV Leiden or FII G20210A mutation and/or with cardiovascular risk factors. Indeed, previous data from an open-label trial showed an elevated number of thrombotic events in IFN-treated patients [31]. Randomized studies are required to determine the incidence of thrombosis in PV and ET patients treated with IFN compared to HU.

Supplementary Materials: The following are available online at http://www.mdpi.com/2072-6694/12/4/992/s1, Table S1: Biological characteristics of ET and PV patients, Table S2: Biological resistance to aspirin according to treatment group.

Author Contributions: Conceptualization, D.F., M.J.-P., C.D. and N.A.; Formal analysis, D.F. and N.A.; Investigation, D.F., L.L., S.L. and B.C.; Resources, M.-G.H., S.A., C.C., C.D. and N.A.; Writing-original draft, D.F., C.D. and N.A.; Writing-review \& editing, D.F., M.-C.B., Y.B., J.D., M.J.-P., C.D. and N.A. All authors have read and agreed to the published version of the manuscript.

Funding: This research was funded by the Agence nationale de la recherche (ANR), grant number ANR-14-CE35-0022-02, ANR-DFG JAKPOT. The APC was funded by the Nouvelle Société Francophone d'Athérosclérose (NSFA).

Acknowledgments: This study was supported by research grants from ANR-DFG JAKPOT (N ANR-14-CE35-0022-02), INSERM. The authors thank Yael Baudouin, Catherine Lacombe, Véronique Morin, Cécile Clément, Laurence Van Vetteren, Fabienne Gaucher, Julie Champoiseau, Carole Gosselin and Valérie Vassou for technical assistance. We are also grateful to the French Intergroup Myeloproliferative (FIM).

Conflicts of Interest: The authors declare no competing financial interests.

\section{References}

1. James, C.; Ugo, V.; Le Couédic, J.-P.; Staerk, J.; Delhommeau, F.; Lacout, C.; Garçon, L.; Raslova, H.; Berger, R.; Bennaceur-Griscelli, A.; et al. A unique clonal JAK2 mutation leading to constitutive signalling causes polycythaemia vera. Nature 2005, 434, 1144-1148. [CrossRef] [PubMed]

2. Pikman, Y.; Lee, B.H.; Mercher, T.; McDowell, E.; Ebert, B.L.; Gozo, M.; Cuker, A.; Wernig, G.; Moore, S.; Galinsky, I.; et al. MPLW515L is a novel somatic activating mutation in myelofibrosis with myeloid metaplasia. PLoS Med. 2006, 3, e270. [CrossRef] [PubMed]

3. Klampfl, T.; Gisslinger, H.; Harutyunyan, A.S.; Nivarthi, H.; Rumi, E.; Milosevic, J.D.; Them, N.C.C.; Berg, T.; Gisslinger, B.; Pietra, D.; et al. Somatic mutations of calreticulin in myeloproliferative neoplasms. N. Engl. J. Med. 2013, 369, 2379-2390. [CrossRef]

4. Casini, A.; Fontana, P.; Lecompte, T.P. Thrombotic complications of myeloproliferative neoplasms: Risk assessment and risk-guided management. J. Thromb. Haemost. 2013, 11, 1215-1227. [CrossRef] [PubMed]

5. Falanga, A.; Marchetti, M. Thrombotic disease in the myeloproliferative neoplasms. Hematol. Am. Soc. Hematol. Educ. Program 2012, 2012, 571-581. [CrossRef]

6. Guy, A.; Gourdou-Latyszenok, V.; Le Lay, N.; Peghaire, C.; Kilani, B.; Vieira Dias, J.; Duplaa, C.; Renault, M.-A.; Denis, C.; Villeval, J.L.; et al. Vascular endothelial cell expression of JAK2V617F is sufficient to promote a pro-thrombotic state due to increased P-selectin expression. Haematologica 2018, 104, 70-81. [CrossRef]

7. Barbui, T.; Finazzi, G.; Falanga, A. Myeloproliferative neoplasms and thrombosis. Blood 2013, 122, $2176-2184$. [CrossRef]

8. Tefferi, A.; Barbui, T. New and treatment-relevant risk stratification for thrombosis in essential thrombocythemia and polycythemia vera. Am. J. Hematol. 2015, 90, 683-685. [CrossRef]

9. Carobbio, A.; Thiele, J.; Passamonti, F.; Rumi, E.; Ruggeri, M.; Rodeghiero, F.; Randi, M.L.; Bertozzi, I.; Vannucchi, A.M.; Antonioli, E.; et al. Risk factors for arterial and venous thrombosis in WHO-defined essential thrombocythemia: An international study of 891 patients. Blood 2011, 117, 5857-5859. [CrossRef] 
10. Marchioli, R.; Finazzi, G.; Specchia, G.; Cacciola, R.; Cavazzina, R.; Cilloni, D.; De Stefano, V.; Elli, E.; Iurlo, A.; Latagliata, R.; et al. Cardiovascular events and intensity of treatment in polycythemia vera. N. Engl. J. Med. 2013, 368, 22-33. [CrossRef]

11. Kiladjian, J.-J.; Giraudier, S.; Cassinat, B. Interferon-alpha for the therapy of myeloproliferative neoplasms: Targeting the malignant clone. Leukemia 2016, 30, 776-781. [CrossRef] [PubMed]

12. Prendergast, Á.M.; Kuck, A.; Van Essen, M.; Haas, S.; Blaszkiewicz, S.; Essers, M.A.G. IFN $\alpha$-mediated remodeling of endothelial cells in the bone marrow niche. Haematologica 2017, 102, 445-453. [CrossRef] [PubMed]

13. Brusson, M.; De Grandis, M.; Cochet, S.; Bigot, S.; Marin, M.; Leduc, M.; Guillonneau, F.; Mayeux, P.; Peyrard, T.; Chomienne, C.; et al. Impact of hydroxycarbamide and interferon- $\alpha$ on red cell adhesion and membrane protein expression in polycythemia vera. Haematologica 2018, 103, 972-981. [CrossRef] [PubMed]

14. Baxter, E.J.; Scott, L.M.; Campbell, P.J.; East, C.; Fourouclas, N.; Swanton, S.; Vassiliou, G.S.; Bench, A.J.; Boyd, E.M.; Curtin, N.; et al. Acquired mutation of the tyrosine kinase JAK2 in human myeloproliferative disorders. Lancet 2005, 365, 1054-1061. [CrossRef]

15. Gisslinger, H.; Zagrijtschuk, O.; Buxhofer-Ausch, V.; Thaler, J.; Schloegl, E.; Gastl, G.A.; Wolf, D.; Kralovics, R.; Gisslinger, B.; Strecker, K.; et al. Ropeginterferon alfa-2b, a novel IFN $\alpha-2 b$, induces high response rates with low toxicity in patients with polycythemia vera. Blood 2015, 126, 1762-1769. [CrossRef] [PubMed]

16. Decker, T.; Müller, M.; Stockinger, S. The yin and yang of type I interferon activity in bacterial infection. Nat. Rev. Immunol. 2005, 5, 675-687. [CrossRef] [PubMed]

17. Niessner, A.; Shin, M.S.; Pryshchep, O.; Goronzy, J.J.; Chaikof, E.L.; Weyand, C.M. Synergistic proinflammatory effects of the antiviral cytokine interferon-alpha and Toll-like receptor 4 ligands in the atherosclerotic plaque. Circulation 2007, 116, 2043-2052. [CrossRef]

18. Lethagen, S.; Widell, A.; Berntorp, E.; Verbaan, H.; Lindgren, S. Clinical spectrum of hepatitis C-related liver disease and response to treatment with interferon and ribavirin in haemophilia or von Willebrand disease. Br. J. Haematol. 2001, 113, 87-93. [CrossRef]

19. Savage, B.; Saldívar, E.; Ruggeri, Z.M. Initiation of platelet adhesion by arrest onto fibrinogen or translocation on von Willebrand factor. Cell 1996, 84, 289-297. [CrossRef]

20. Arellano-Rodrigo, E.; Alvarez-Larrán, A.; Reverter, J.-C.; Colomer, D.; Villamor, N.; Bellosillo, B.; Cervantes, F. Platelet turnover, coagulation factors, and soluble markers of platelet and endothelial activation in essential thrombocythemia: Relationship with thrombosis occurrence and JAK2 V617F allele burden. Am. J. Hematol. 2009, 84, 102-108. [CrossRef]

21. Panova-Noeva, M.; Marchetti, M.; Spronk, H.M.; Russo, L.; Diani, E.; Finazzi, G.; Finazzi, G.; Salmoiraghi, S.; Rambaldi, A.; Rambaldi, A.; et al. Platelet-induced thrombin generation by the calibrated automated thrombogram assay is increased in patients with essential thrombocythemia and polycythemia vera. Am. J. Hematol. 2011, 86, 337-342. [CrossRef] [PubMed]

22. Marchetti, M.; Castoldi, E.; Spronk, H.M.H.; Van Oerle, R.; Balducci, D.; Barbui, T.; Rosing, J.; Ten Cate, H.; Falanga, A. Thrombin generation and activated protein $\mathrm{C}$ resistance in patients with essential thrombocythemia and polycythemia vera. Blood 2008, 112, 4061-4068. [CrossRef]

23. Duchemin, J.; Ugo, V.; Ianotto, J.-C.; Lecucq, L.; Mercier, B.; Abgrall, J.-F. Increased circulating procoagulant activity and thrombin generation in patients with myeloproliferative neoplasms. Thromb. Res. 2010, 126, 238-242. [CrossRef] [PubMed]

24. Dielis, A.W.J.H.; Castoldi, E.; Spronk, H.M.H.; Van Oerle, R.; Hamulyák, K.; Ten Cate, H.; Rosing, J. Coagulation factors and the protein $\mathrm{C}$ system as determinants of thrombin generation in a normal population. J. Thromb. Haemost. 2008, 6, 125-131. [CrossRef] [PubMed]

25. Tripodi, A.; Chantarangkul, V.; Gianniello, F.; Clerici, M.; Lemma, L.; Padovan, L.; Gatti, L.; Mannucci, P.M.; Peyvandi, F. Global coagulation in myeloproliferative neoplasms. Ann. Hematol. 2013, 92, 1633-1639. [CrossRef] [PubMed]

26. Best, D.; Senis, Y.A.; Jarvis, G.E.; Eagleton, H.J.; Roberts, D.J.; Saito, T.; Jung, S.M.; Moroi, M.; Harrison, P.; Green, F.R.; et al. GPVI levels in platelets: Relationship to platelet function at high shear. Blood 2003, 102, 2811-2818. [CrossRef]

27. Ugo, V.; Tondeur, S.; Menot, M.-L.; Bonnin, N.; Le Gac, G.; Tonetti, C.; Mansat-De Mas, V.; Lecucq, L.; Kiladjian, J.-J.; Chomienne, C.; et al. Interlaboratory development and validation of a HRM method applied to the detection of JAK2 exon 12 mutations in polycythemia vera patients. PLoS ONE 2010, 5, e8893. [CrossRef] 
28. Cassinat, B.; Verger, E.; Kiladjian, J.-J. Interferon alfa therapy in CALR-mutated essential thrombocythemia. N. Engl. J. Med. 2014, 371, 188-189. [CrossRef]

29. Ajzenberg, N.; Aubry, P.; Huisse, M.-G.; Cachier, A.; El Amara, W.; Feldman, L.J.; Himbert, D.; Baruch, D.; Guillin, M.-C.; Steg, P.G. Enhanced shear-induced platelet aggregation in patients who experience subacute stent thrombosis: A case-control study. J. Am. Coll. Cardiol. 2005, 45, 1753-1756. [CrossRef]

30. Ollivier, V.; Wang, J.; Manly, D.; Machlus, K.R.; Wolberg, A.S.; Jandrot-Perrus, M.; Mackman, N. Detection of endogenous tissue factor levels in plasma using the calibrated automated thrombogram assay. Thromb. Res. 2010, 125, 90-96. [CrossRef]

31. Masarova, L.; Patel, K.P.; Newberry, K.J.; Cortes, J.; Borthakur, G.; Konopleva, M.; Estrov, Z.; Kantarjian, H.; Verstovsek, S. Pegylated interferon alfa-2a in patients with essential thrombocythaemia or polycythaemia vera: A post-hoc, median 83 month follow-up of an open-label, phase 2 trial. Lancet Haematol. 2017, 4, e165-e175. [CrossRef]

(C) 2020 by the authors. Licensee MDPI, Basel, Switzerland. This article is an open access article distributed under the terms and conditions of the Creative Commons Attribution (CC BY) license (http://creativecommons.org/licenses/by/4.0/). 